\title{
Carcinoma adenoneuroendocrino mixto de la ampolla de Vater: reporte de caso
}

\author{
Carlos Paredes M. ${ }^{1}$, Augusta Velasco A. ${ }^{2}$ y Xavier Wong-Achi ${ }^{2}$
}

\section{Mixed adenoneuroendocrine carcinoma of the ampulla of Vater: Case report}

Introduction: Mixed adenoneuroendocrine carcinomas (MANEC) are types of biphasic tumors, morphologically recognized in the presence of a neoplastic formation constituted simultaneously by glandular epithelium and neuroendocrine cells. Only 19 cases located in the ampulla of Vater have been reported in the literature. Within the gastrointestinal tract, these neoplasms predominate in the stomach or colon. Aim: Report a case of MANEC; review of the epidemiology, prognosis and treatment of these tumors. Materials and Method: Case presentation of a patient diagnosed with mixed adeno-neuroendocrine carcinoma of the ampullary region. Discussion: The clinical presentation, management and prognosis are similar to ampullary adenocarcinoma. These tumors are diagnosed with a histopathological examination of the resected specimen. Both components must be histologically malignant, and each of them must represent at least $30 \%$ of the lesion. Conclusion: MANEC of the ampulla are rare tumors worldwide, being this case the first reported in our institute.

Key words: duodenal neoplasms; ERCP; adenoneuroendocrine carcinoma; pancreaticoduodenectomy.

\section{Resumen}

Introducción: Los carcinomas adenoneuroendocrinos mixtos (MANEC) son tipos de tumores bifásicos, reconocidos morfológicamente ante la presencia de una formación neoplásica constituida de manera simultánea por epitelio glandular y células neuroendocrinas. Dentro del tracto gastrointestinal, estas neoplasias predominan en el estómago o el colon. Solo 19 casos localizados en la ampolla de Vater han sido reportados por la literatura. Objetivo: Reportar un caso de MANEC; revisar la epidemiología, pronóstico y tratamiento de estos tumores. Materiales y Método: Presentación de caso clínico de una paciente con diagnóstico de adenocarcinoma neuroendocrino mixto de la región ampular. Discusión: La presentación clínica, el manejo y el pronóstico son similares al del adenocarcinoma ampular. Se diagnostican con el examen histopatológico de la muestra resecada. Ambos componentes deben ser histológicamente malignos, y cada uno de ellos debe representar al menos el 30\% de la lesión. Conclusión: Los MANEC ampulares son tumores poco comunes a nivel mundial, siendo éste el primer caso reportado en nuestro instituto. Palabras clave: neoplasias duodenales; CPRE; carcinoma adenoneuroendocrino; pancreatoduodenectomía.

\section{Introducción}

El carcinoma adenoneuroendocrino mixto (MANEC, por sus siglas en inglés) es una patología rara. La primera descripción de un tumor gastrointestinal con componentes exocrinos y neuroendocrinos fue publicado por Cordier en $1924^{1}$. Desde entonces, pocos casos han sido reportados en la literatura. En 1987 Lewin sugirió la clasificación de este tipo de neoplasias en tres subtipos diferentes ${ }^{2}$, y en 2010 la OMS publicó una nueva clasificación basada en el índice mitótico y cuantificación de $\mathrm{Ki} 67^{3,4}$.

Se presentan con mayor frecuencia en pacientes añosos y de género masculino ${ }^{2}$. Estos debutan sin sintomatología específica ni imágenes características, siendo mayormente diagnosticados de manera incidental como hallazgo posterior a estudios histopatológicos e inmunohistoquímicos. En cuanto a localización, según los registros se ha observado mayor incidencia en estómago, vesícula, páncreas y colon, siendo la localización en duodeno muy $\operatorname{rara}^{4,5}$.

La aplicación sistemática de inmunohistoquímica
'Hospital Móvil de Guayaquil. Guayaquil, Ecuador. Instituto Oncológico Nacional "Dr. Juan Tanca Marengo" ION-SOLCA. Guayaquil, Ecuador.

Recibido el 22 de julio de 2018, aceptado para publicación el 17 de agosto de 2018.

Correspondencia a: Dr. Xavier Wong A. xawong@uees.edu.ec 


\section{CASOS CLÍNICOS}

Figura 1. Colangiopancreatografía retrógrada endoscópica.

Figura 2. Posterior a esfinterotomía selectiva y toma de muestras, se realiza colocación de stent metálico.

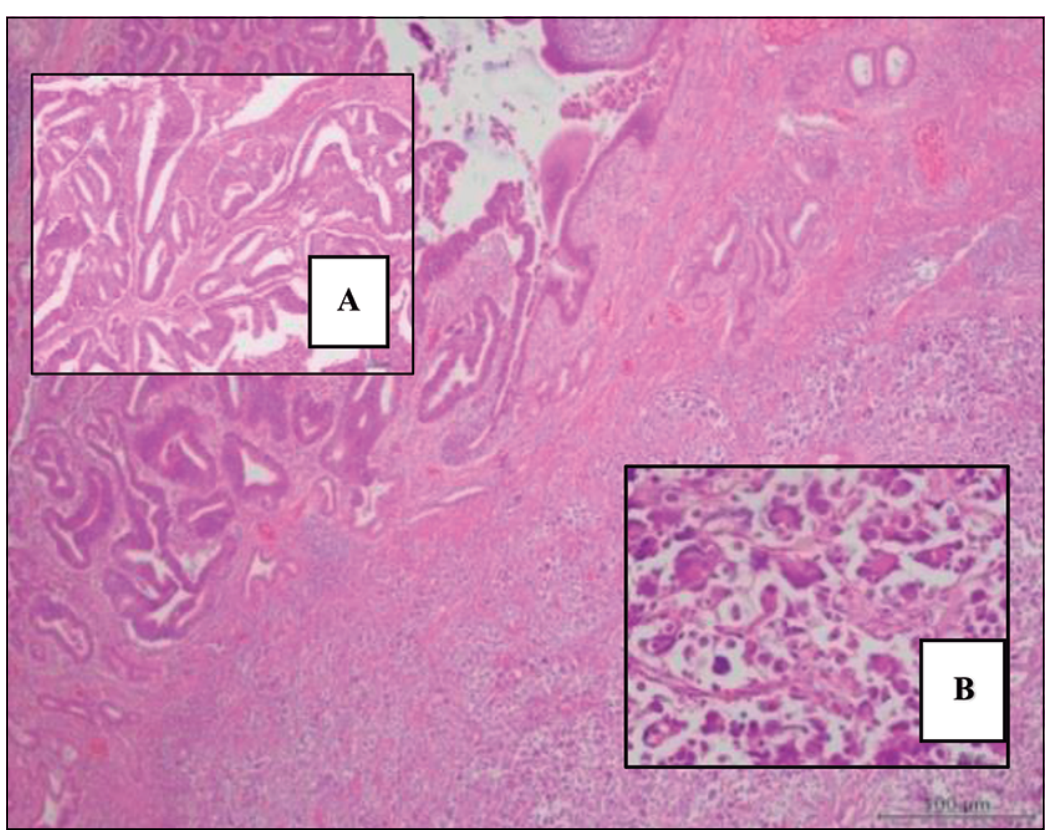

Figura 3. Visión microscópica con tinción de hematoxilina eosina. H/E 4x, tumor adenoneuroendocrino mixto. A: 10x, células neoplásicas formando cordones y luces glandulares. B: 40x, área poco diferenciada de células neoplásicas con núcleos pleomórficos, hipercromáticos con patrón sincitial. ha permitido establecer la presencia de componentes celulares neuroendocrinos en neoplasias exocrinas.

\section{Materiales y Método}

Descripción de un caso clínico y revisión bibliográfica sobre el tema. La información fue obtenida a partir de la historia clínica del paciente, revisión y análisis de la literatura publicada mediante el uso de motores de búsqueda y base de datos del registro de tumores del Instituto Oncológico Nacional "Dr. Juan Tanca Marengo" ION-SOLCA de la ciudad de Guayaquil, Ecuador.

\section{Caso clínico}

Paciente de género femenino de 60 años de edad sin antecedentes de relevancia, acude a la consulta por dolor abdominal en hipocondrio derecho, alza térmica e ictericia, acompañada de coluria y acolia. Datos de laboratorio: leucocitos $12.000 \mathrm{u} / \mathrm{l}$; neutrófilos: $80 \%$; bilirrubina total: $15,6 \mathrm{mg} / \mathrm{dl}$; bilirrubina conjugada: $11,4 \mathrm{mg} / \mathrm{dl}$; amilasa: $126 \mathrm{mg} / \mathrm{dl}$; lipasa: $81 \mathrm{mg} / \mathrm{dl}$.

Se le realiza a la paciente colangiopancreatografía retrógrada endoscópica (Figura 1) donde se evidenció lesión neoplásica en ampolla de Vater de $3 \times 2 \mathrm{~cm}$. Se procedió con esfinterotomía selectiva, con toma de muestra para biopsia y posterior colocación de stent metálico en conducto colédoco (Figura 2). Informe patológico posterior a revisión de bloques y placas: adenocarcinoma de tipo intestinal, sobre adenoma velloso. La paciente es sometida a pancreatoduodenectomía por técnica de Whipple, cursando postquirúrgico sin complicaciones.

Según estudio patológico, se evidencia en la macroscopía ampolla de Vater engrosada, obstruida por lesión redondeada de 2,3 x 1,8 cm. El diagnóstico es confirmado por histopatología e inmunohistoquímica: presentando $35 \%$ de componente neuroendocrino y $65 \%$ de componente adenocarcinoma (Figura 3). Por inmunohistoquímica, el componente neuroendocrino resultó positivo a Synaptofisina, CD56 $\mathrm{y}$ focalmente para cromogranina-A, y negativo en el componente adenocarcinoma. Además, el Ki67 dio un porcentaje de $80 \%$ en el componente neuroendocrino (clasificándolo así como de alto grado) y $65 \%$ en el componente adenocarcinoma (Figura 4). Diagnóstico final: adenocarcinoma neuroendocrino mixto tipo colisión, según la clasificación propuesta por Lewin. La estadificación tumoral patológica 


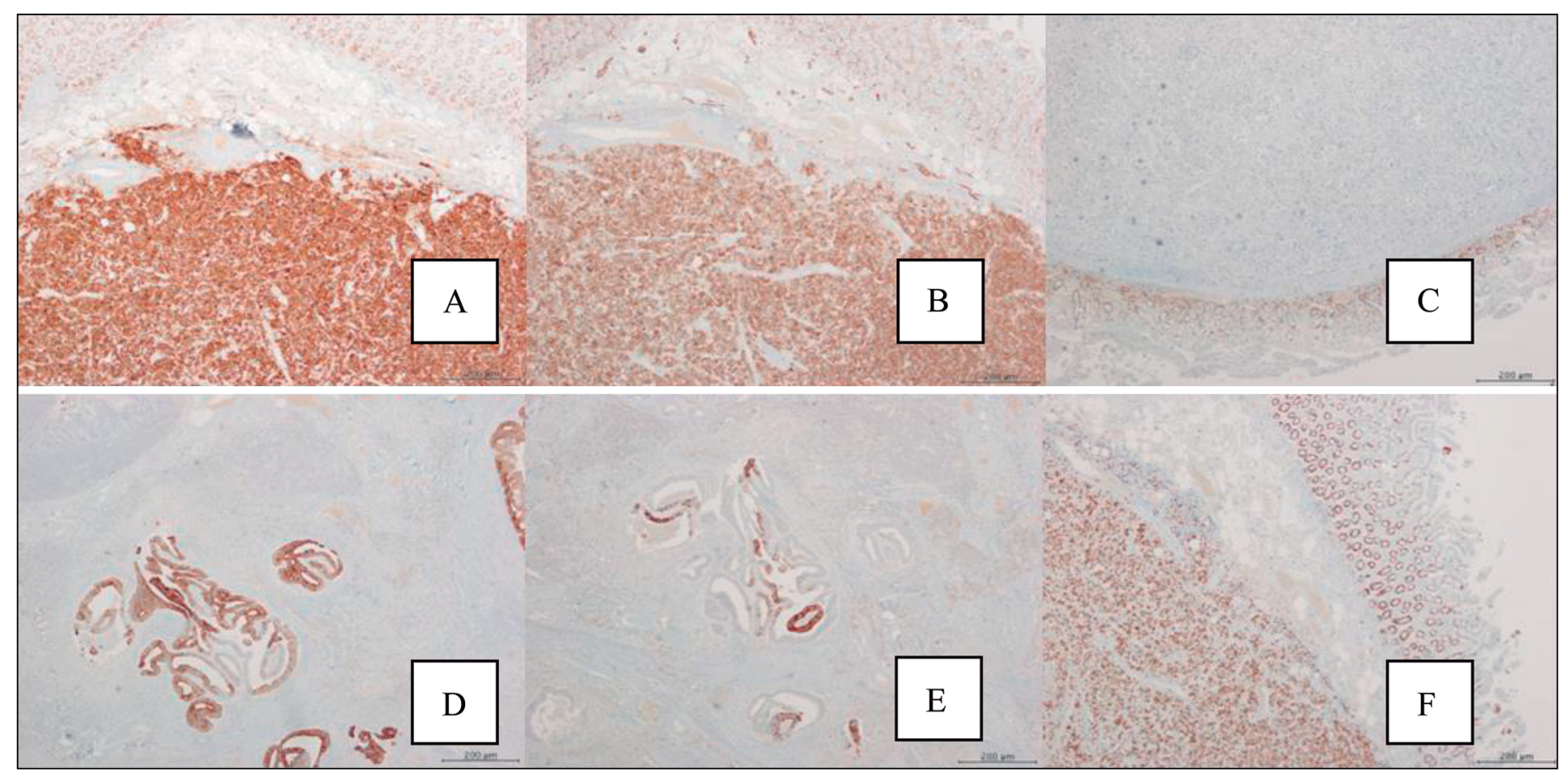

Figura 4. A: $4 x$, tinción de inmunohistoquímica de Synaptofisina, el componente de carcinoma neuroendocrino muestra una fuerte positividad. B: 4x, la tinción de inmunohistoquímica muestra que células de carcinoma neuroendocrino son positivas para CD56. C: 4x, tinción de inmunohistoquímica de cromogranina-A, muestra positividad focal y difusa en células de carcinoma neuroendocrino. D-E: $4 x$, tinción de inmunohistoquímica de CK7 y CK20, moderada y difusa positividad citoplasmática de células de adenocarcinoma. F: 4x, tinción para Ki67 muestra en las células del carcinoma neuroendocrino un índice de proliferación alto (80\%).

fue de T3N0M0. En la actualidad, la paciente cursa clínicamente estable, sin evidencia de recidiva de la enfermedad.

\section{Discusión}

En el MANEC se presentan los componentes de tumor neuroendocrino y de adenocarcinoma en diferente distribución; según la clasificación de la OMS 2010, se requiere que los componentes, tanto neuroendocrino como glandular exocrino, representen más del $30 \%$ de la constitución del tumor para ser denominado adenocarcinoma neuroendocrino mixto ${ }^{2}$. En el presente caso el componente de adenocarcinoma representaba el $65 \%$ del tumor. De hecho, hay mucha variación en el porcentaje de componentes entre el adenocarcinoma y el tumor neuroendocrino. Sin embargo, aquellos que se ajustan a los criterios de MANEC son infrecuentes, y solo se pueden encontrar informes de casos en la literatura, y por lo tanto, nuestro conocimiento es inadecuado. Lewin ${ }^{3}$ describió varios subtipos; de éstos, la variante compuesta o combinada es carac- terizada por presentar su estructura celular mezclada dentro de la misma lesión; la de colisión se presenta como dos estructuras histológicas separadas dentro del mismo tejido, y en la variante anficrina se observa que las células muestran una diferenciación bivalente (características neuroendocrina y adenocarcinomatoidea) dentro de su citoplasma. De acuerdo con este esquema de subtipos, el caso que presentamos fue considerado como un carcinoma tipo colisión.

Acorde a la literatura revisada, los pacientes tienden a cursar con sintomatología inespecífica como dolor abdominal, náuseas y vómitos; el caso reportado debutó con clínica que sugería un cuadro colestásico. El diagnóstico por imágenes es de mayor importancia, la endoscopía nos permite realizar toma de biopsia. A su vez estudios como tomografía computarizada, resonancia magnética o ecografía brindan información útil. El diagnóstico definitivo se realiza a través del estudio histopatológico, así como el inmunohistoquímico posterior a la cirugía ${ }^{6}$. En la inmunohistoquímica para confirmar el componente neuroendocrino se utiliza la tinción para cromogranina A, Synaptofisina, CD56 y la enolasa, 
siendo necesario el resultado positivo de dos de las mismas para su diagnóstico ${ }^{2}$. En nuestro caso, se evidenció positividad para synaptofisina y CD56, mientras la cromogranina-A resultó positiva focalmente, confirmándose el diagnóstico de MANEC. La estadificación del tumor depende del tamaño y extensión del mismo, la invasión ganglionar y de la diseminación a otros órganos. Mientras que la determinación del grado histológico se basa en el conteo mitótico y el índice de Ki67.

El tratamiento se basa en la cirugía, siendo la pancreatoduodenectomía el procedimiento óptimo para carcinomas localizados en la ampolla de Vater. Sin embargo, para pacientes que no puedan tolerar la escisión radical, la escisión local puede ser una opción válida. Posterior a esto se debe adoptar un tratamiento multimodal con radioterapia adyuvante y/o quimioterapia ${ }^{7,8}$.

El MANEC de la ampolla de Vater es un subtipo raro de tumor ampular con comportamiento agresivo y de mal pronóstico. La serie más grande publicada de 2.564 adenocarcinomas periampulares resecados mostró una supervivencia media estimada de 22 meses ${ }^{9}$, sin embargo, no menciona la presencia de tumores mixtos con componente neuroendocrino, por lo que la cifra de supervivencia media no se puede aplicar al caso clínico específicamente. En otros reportes la supervivencia en 5 años varía de 33 a $53 \%{ }^{10}$. A su vez, el compromiso de ganglios linfáticos, los márgenes quirúrgicos positivos, el estadio avanzado y tumores poco diferenciados se asocian con una supervivencia significativamente menor después de la resección quirúrgica ${ }^{11,12}$. Varios autores han sugerido que el pronóstico debe depender principalmente del estadio del adenocarcinoma, mientras que otros sugieren que el tumor neuroendocrino tiene una mayor capacidad de invasión y, por lo tanto, afecta más al pronóstico que el adenocarcinoma. Creemos razonable tratar según el componente más agresivo del tumor ${ }^{13,14}$. En la actualidad, la paciente del presente caso cursa el $6^{\circ}$ mes postquirúrgico estable, en seguimiento por la consulta externa.

\section{Conclusión}

El MANEC es un tumor de baja incidencia a nivel mundial, y puede causar errores de diagnóstico si no se sospecha del mismo. La sintomatología es inespecífica; las imágenes revelan la presencia del tumor y permiten la toma de muestra para biopsia. La histopatología e inmunohistoquímica permiten dar un diagnóstico preciso y confiable. El tratamiento es quirúrgico y debe estar enfocado hacia el componente de pobre diferenciación y mayor agresividad. La epidemiología de estos tumores en nuestro medio no ha sido analizada, es necesario el diseño de estudios que permitan establecer su prevalencia, así como el seguimiento y tiempo de sobrevida de los pacientes posterior al tratamiento quirúrgico.

\section{Responsabilidades éticas}

Protección de personas y animales: Los autores declaran que para esta investigación no se han realizado experimentos en seres humanos ni en animales.

Confidencialidad de los datos: Los autores declaran que han seguido los protocolos de su centro de trabajo sobre la publicación de datos de pacientes.

Derecho a la privacidad y consentimiento informado: Los autores declaran que en este artículo no aparecen datos de pacientes.

Conflictos de interés: No hay.

\section{Referencias}

1. Cordier R. Les cellules argentaffines dans les tumeurs intestinalis. Arch Int Med Exp. 1924;1:59-63.

2. Zhen H, Wei-Dong X, Yong L, Song H, Jun C, Jian A. Mixed adenoneuroendocrine carcinoma of the ampulla: Two case reports. World J Gastroenterol. 2015;21:2254-9.

3. La Rosa S, Marando A, Sessa F, Capella C. Mixed Adenoneuroendocrine Carcinomas (MANECs) of the Gastrointestinal Tract: An Update.
Cancers 2012;4:11-30.

4. Minaya A, García J, Mendoza F, Noguerales F, Granell J. Large cell neuroendocrine-adenocarcinona mixed tumour of colon: Collision tumour with peculiar behaviour. What do we know about these tumours? Annals of Medicine and Surgery 2015;4;399:403.

5. Rindi G, Petrone G, Inzani F. The 2010 WHO classification of digestive neuroendocrine neoplasms: a critical appraisal four years after its introduction. Endocr Pathol. 2014;25:186-92.

6. Jain A, Singla S, Jagdeesh
KS, Vishnumurthy HY. Mixed adenoneuroendocrine carcinoma of cecum: A rare entity. J Clin Imaging Sci. 2013;3:10

7. Kwok CM. Mixed Adenoneuroendocrine Carcinoma of the Stomach. Case Rep Gastroenterol. 2015;9:241-5.

8. Acosta A, Wiley E. Primary Biliary Mixed Adenoneuroendocrine Carcinoma (MANEC). Arch Pathol Lab Med. 2016;140:1157-62.

9. He J, Ahuja N, Makary MA, Cameron JL, Eckhauser FE, Choti MA, et al. 2564 resected periampullary adenocarcinomas 
at a single institution: trends over three decades. HPB (Oxford) 2014;16:83-90.

10. Onishi I, Kitagawa H, Harada K, Maruzen S, Sakai S, Makino I, et al. Intraductal papillary neoplasm of the bile duct accompanying biliary mixed adenoneuroendocrine carcinoma. World J Gastroenterol. 2013;19:3161-4.

11. Westgaard A, Tafjord S, Farstad IN, Cvancarova M, Eide TJ, Mathisen O, et al. Pancreatobiliary versus intestinal histologic type of differentiation is an independent prognostic factor in resected periampullary adenocarcinoma. BMC Cancer 2008;8:170. doi: 10.1186/14712407-8-170.

12. Klein F, Jacob D, Bahra M, Pelzer U, Puhl G, Krannich A, et al. Prognostic factors for long-term survival in patients with ampullary carcinoma: the results of a 15-year observation period after pancreaticoduodenectomy. HPB Surg. 2014;2014:970234. doi: $10.1155 / 2014 / 970234$.
13. Mahansaria S, Agrawal N, Arora A, Bihari C, Appukuttan M, Chattopadhyay T. Ampullary Mixed Adenoneuroendocrine Carcinoma: Surprise Histology, Familiar Management. Int J Surg Pathol 2017;25:585-91. doi: 10.1177/1066896917712454.

14. Max N, Rothe A, Langner C. Mixed adenoneuroendocrine carcinoma of the ampulla of Vater: A case report. Mol Clin Oncol. 2016;5:95-98. DOI: 10.3892/ mco.2016.858. 